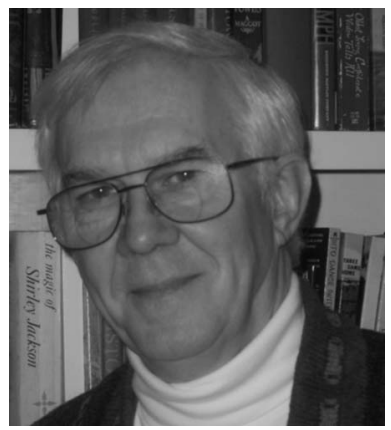

\section{Of Macs and Maps}

This editorial is being written on my new Mac computer. It is one of many that I have had over the years. I have no wish to engage in one of the technotheological battles that seem to erupt once someone has declared his or her colors (Mac or PC). Rather, I want to consider what my Macs have enabled me to do during the 25 years that they have been available.

Actually, our family started with an Apple II soon after it was introduced in 1977. Before we bought one, I spent several weeks trying to handicap the computer race. I would go into a computer store and try all the brands that were on the market, read all the early computer magazines, then go back again. It may have been the fact that you could use a small color TV as a monitor with the Apple that caused me to buy one. Or it might have been because the kids could jump right in and use it. I ordered it before we left to go to the beach for our summer vacation. We returned to Atlanta and before going home, we picked up the machine and an additional $48 \mathrm{~KB}$ of RAM for $\$ 80$. (Wow! A whole $64 \mathrm{~K}$ of memory!) Once at home the kids (including me) immediately loaded the programs on tape cassettes that came with the machine and ran them that weekend. (I still have the cassettes of Lemonade Stand and Brian's Theme.) The next week we wrote our own programs.

I wrote a Jack-o'-lantern program that we ran with the monitor in the window during Halloween. I collaborated with one of Ronchi's students on a ray trace program, EZ-ray. (The first thing I did was to write a missing Seidel coefficients routine for it.) I wrote some small programs for Apple magazines and made just enough money to be able to deduct the machine on my taxes. I was beginning to write a movable map routine, which used the map from The Lord of the Rings to display parts of Middle-earth, when the first Macintosh appeared. I bought an early model and lugged it out to Arizona to the Optical Sciences Center where I spent an academic year with Bob Shannon and Stacey Dereniak. I taught myself how to use MacDraw to make electronic circuit patterns (maps, as it were) for some early scanning tunneling microscope experiments at Arizona.

When I took my next sabbatical at the University of Oulu in Finland, I was lugging a Mac portable. It was so heavy (over $7 \mathrm{~kg}$ ) that your feet would go to sleep if you tried to use it as a laptop. E-mail was just becoming a conventional way of communicating, so I used it to keep in touch with my family in Atlanta. During that fall the Atlanta Braves went to the World Series. I would get up in the middle of the night and watch the game on EuroSport. Between innings Helen and I would engage in e-mail discussions of the game in progress. My Mac portable also proved its usefulness when an attempted coup of the Russian government took place in Moscow in 1991, just six weeks before the Education in Optics Conference was to be held in Leningrad. Using the Mac, I kept in touch with SPIE's conference staff. I was able to follow the deliberations on whether there would be a conference from Finland. In the end, it took place with no major events. Well, there was one. Having arrived in Leningrad, we left by way of St. Petersburg. During our stay the Russians changed the name of the city back to that of its Tsarist past.

Since that time I have gone through quite a few Macs, each a little more powerful and colorful than before. Despite having participated in this incremental progress in computing, I am still amazed by what I can do with them. I can visit our granddaughters using an iSight camera on a regular basis ("Grandparent Technology," March 2004). I can correct, compose, and publish my travel pictures on the Web and in a photobook that I designed. I can publish a textbook that my wife has written ("Self-Publishing," June 2008). I can edit this journal from a study while I keep an eye on our garden.

And now, with this 30 -in. screen that displays these words, I can write a book using a word processor, generate ray traces and lens analysis on Code V, and incorporate all of this into the book that I can compose exactly as I want it to look. (I am a control freak when it comes to graphic design.) And all of the work is visible and accessible from the same screen.

Today, I was settling into my new system, assigning papers for Optical Engineering, and taking care of busi- 
ness. One of the items in my to-do list was to map out a trip to visit some rhododendron nurseries around Athens, Georgia. I had already determined the best nearby nurseries and placemarked them in Google Earth on my old Mac. So when I opened Google Earth on this new Mac to get the lay of the land (literally), I was startled. The image of earth on that 30-in. screen expanded, rotated, and then zoomed in on east Georgia. Wow! The amount of territory that I could see and comprehend was overwhelming. Granted, we have a large LCD television and it would seem that the display of a patchy mosaic of maps would not be impressive, but it is. Having your nose less than two feet away from four million pixels displaying a familiar map is an experience! In thirty years' time I came from trying to create a panoramic map of a fantastic land to observing a fantastic panorama of a map of a familiar landscape.

What remains after my amazement is the realization that much of the progress that I have witnessed in that time was due to the work of many optical engineers and scientists. Without the advances in display technology that produced the plasma and liquid crystal displays, without the enormous work that has been put into image processing and compression, and without the optical fiber technology that permits DSLs to push all that information into my Mac, I would be looking at a huge glorified cathode ray tube whose resolution and color rendition would never draw a gasp of delight. The ingenuity of my colleagues in optical engineering is pretty much taken for granted. It is the display of familiar information in a dynamic and beautiful manner that amazes me and makes me appreciate what our field has helped to accomplish.

Donald C. O'Shea Editor 